\title{
Dysbiosis of the Oral Microbiome: Association with and Prevention of Human Diseases
}

\author{
Zhongwei Zhang1, Wei Yang1,2, Stanley T. Omaye ${ }^{3}$ \\ ${ }^{1}$ School of Public Health, Nanchang University, Nanchang, China \\ ${ }^{2}$ School of Community Health Sciences, University of Nevada, Reno, USA \\ ${ }^{3}$ Department of Nutrition, University of Nevada, Reno, USA \\ Email: redmaple9966@163.com,weiyang@unr.edu, omaye@unr.edu
}

How to cite this paper: Zhang, Z.W., Yang, W. and Omaye, S.T. (2019) Dysbiosis of the Oral Microbiome: Association with and Prevention of Human Diseases. Food and Nutrition Sciences, 10, 793-814. https://doi.org/10.4236/fns.2019.107058

Received: June 6, 2019

Accepted: July 26, 2019

Published: July 29, 2019

Copyright () 2019 by author(s) and Scientific Research Publishing Inc. This work is licensed under the Creative Commons Attribution International License (CC BY 4.0).

http://creativecommons.org/licenses/by/4.0/

\section{(c) (i) Open Access}

\begin{abstract}
Oral diseases are associated with systemic diseases; such as type II diabetes, cardiovascular disease, rheumatoid arthritis (RA), and neurological diseases. Coincidentally, the oral microbiome (fluids or extracts) is readily accessible and easily sampled; therefore, serving as a diagnostic or prognostic tool for health status. The oral microbiome is a useful research model for studying fundamental questions of the human microbiome. In this narrative literature review, we examine the characteristics of oral microorganisms, the relationship between oral microorganisms and human diseases, and the important role of oral microorganisms in disease prevention. Also, we illustrate the usefulness of sampling the oral microbiome in developing the diagnosis and prognostic treatment strategies for oral and systemic diseases to accelerate their clinical application. Selective saliva biomarkers and microbiome can serve for useful indices to oral diseases and systemic diseases, and as a model research tool, the oral cavity has many uses in the clinical and research environment. The relationships between oral health and systemic diseases are quite profound, and future research will illuminate opportunities for fruitful preventative measures and therapeutics.
\end{abstract}

\section{Keywords}

Oral Microbiome, Chronic Diseases, Dysbiosis, Inflammatory Diseases, Probiotics

\section{Introduction}

The human oral cavity is the beginning part of the digestive tract. Contiguous with the oral cavity are the tonsils, pharynx, esophagus, Eustachian tube, middle ear, trachea, lungs, nasal passages, and sinuses. The oral cavity is the portal for 
the transfer and exchange of environmental substances between the human body and the outside and is the gateway for pathogenic bacteria and toxic substances to invade the body. Also, the oral cavity is one of the most complex, dynamic, and diverse microbial collections in the human body. This collection termed the "oral microbiome"; comprises mainly bacteria, viruses, fungi, protozoa, and archaebacterial [1] [2]. The bacterial communities found in the mouth are highly complex with approximately 1000 species present at different habitats such as saliva, teeth, gingival sulcus, attached gingiva, tongue, cheek, lip, and hard and soft palate. The oral cavity is second only to the colon as the most complex microbial population in the body. In addition, because the liquid or extract of oral microorganism is easy to obtain and sample, the oral microbiome is the most studied human microbial community, and it is used as a prediction or prediction model of general human hesitation state in general [3]. The combination of new generation sequencing technology and information analysis technology has rapidly promoted oral microbiology from low-resolution single species investigation to high dimensional community research. The objective of this narrative literature review is to reveal the effect of oral microorganisms on oral cavity dysbiosis and whole body health, excavating its role in disease diagnosis and treatment prediction, and promoting oral microorganisms important contribution to the process of clinical practice.

\section{Role of the Oral Microbiome in Health}

As an important colonization site of human microorganisms, human oral microorganisms are part of the main research objects of the "National Institutes of Health (NIH) Human Microbiome Project (HMP) (https://hmpdacc.org/hmp/)", which started in 2008. The overall undertaking of the HMP is to produce resources to enable the description of the human microbiota to further our understanding of how the microbiome impacts human health and disease.

In recent years, many studies have shown that the oral microbiome plays an important role in maintaining oral and systemic health [4]. The dysbiosis or ecological imbalance of oral microorganisms cannot only induce many oral diseases (dental caries and periodontal diseases) but can be associated with systemic diseases, such as cancers, type II diabetes, rheumatoid arthritis, cardiovascular disease, etc. Dental diseases have a significant impact on oral health and economic development. According to the report of the International Association for Dental Research and the American Association of Dental Research (IADR/AADR; https://ncdalliance.org/international-association-for-dental-research-iadr), the number of people with untreated oral conditions reached 3.5 billion in 2015 [5]. Untreated caries in permanent teeth was the most prevalent condition around the globe among all 313 diseases assessed in the 2015 Global Burden of Disease (GBD) Study, affecting 2.5 billion people; untreated caries in deciduous teeth affected 573 million children and severe periodontal disease affected 538 million people, leading to 276 million people with total tooth loss worldwide. Within the 
limitations of available data sources, the economic impact of dental diseases in 2015 amounted to $\$ 544.41$ billion, of which $\$ 356.80$ billion were due to direct treatment costs and $\$ 187.61$ billion due to productivity losses. In recent years, with further microbiological research, the association of oral microorganisms and several major chronic diseases has been confirmed, including digestive system disease, cardiovascular disease, cancers, preterm birth, diabetes, rheumatoid arthritis, nervous system diseases, etc. [4]. Therefore, further study of oral microbiome is of great significance to promote the health of all people for social and economic development.

The human oral microbiome is the most studied human microflora because it is easily sampled and is intensely associated with important oral infectious diseases and systemic disease. The National Individual Microbial Group Test Project, launched by the United States Government in 2015

(https://commonfund.nih.gov/hmp/programhighlights), used oral, skin, and intestinal microbial communities as the main monitoring tools. The objective of the project is to transform the research findings from human microbiology to clinical application and to search for more accurate molecular markers for disease the diagnosis from the oral microbiome. Also, the oral cavity microbiome is an important gateway for communication between microbes in the external environment and the human body. Oral microbiology research will be an important area of study between environmental microbiology and human microbiology.

\section{Characteristics of the Oral Microbiome and Its Influencing Factors}

\subsection{Complex Species}

The Human Oral Microbiome Database (HOMD) contains a total of 619 described and curated human oral taxa. Of these, 151 taxa have at least one genomic sequence and annotation available, and an additional 65 have a genome-sequencing project in progress [6]. The complexity of human oral microbiota is far beyond the range of species included in HOMD. Researchers found 318 genera of oral microbes covering 22 taxonomic phyla [7]. Assemblage the sequences in operational taxonomic units (6\%) yielded 3621 and 6888 species-level phyllo types in saliva and plaque, respectively.

\subsection{Multi-Level and Multi-Site Distribution}

The oral cavity contains both exfoliated surface (mucous membrane), non-exfoliated solid surface (tooth surface and denture surface), and flowing saliva. All oral microbiome show a high degree of niche specificity among sample locations. For example, there are significant differences in the composition of dental plaque, mucosal flora, and saliva flora. The microorganism richness of dental plaque colonization is the highest, saliva is second, and buccal mucosa is the lowest [8]. Soft tissues, saliva, and tongue were more often colonized by cariogenic streptococcal species than teeth. In a comparative analysis made on the microorganism 
groups at eight oral sites, including saliva, supragingival plaque, hard palate, palatal tonsil, tongue dorsum, buccal mucosa, subgingival plaque, and keratinized gingiva. The distribution of Corynebacterium varied with the location; most of Corynebacterium martensii was found in supragingival plaque, while, most of Corynebacterium silvery was found in saliva and little found in the hard palate [9]. Investigators used checkerboard DNA-DNA hybridization to further analyze samples from children for bacterial enumeration taken from saliva, supragingival and subgingival plaque, tongue dorsum, and soft tissues, and found soft tissues, saliva and tongue were more often colonized by cariogenic streptococcal species than teeth [10].

\subsection{Age and Replacement Pressure of Dentition}

Research looking at neonates' skin, oral mucosal, and nasopharyngeal aspirate, sampled $<5 \mathrm{~min}$, and meconium sampled $<24 \mathrm{~h}$, after delivery, used multiplexed $16 \mathrm{~S}$ rRNA gene pyrosequencing to characterize bacterial communities from mothers and their newborn babies. The results also display that vaginally delivered infants attained bacterial communities approximating their own mother's vaginal microbiota, controlled by Lactobacillus, Prevotella, or Sneathia spp., and C-section infants harbored bacterial communities comparable to those found on the skin surface, subjugated by Staphylococcus, Corynebacterium, and Propionibacterium spp. Moreover, in contrast to their mothers, the newborns protected bacterial communities that were, in essence, undifferentiated across the skin, oral, nasopharyngeal, and gut habitats irrespective of delivery mode [11]. This illustrates that the time and process of microbial differentiation in different parts of the body are worthy of further study.

Structure of oral microbiota can differ substantially among distinct age groups. Evidence has recently arisen that changes in the human microbiota continue throughout the human life cycle. The growth of the oral microbiota involved profound alterations in diversity and composition that took place not merely over the first three postnatal years, but also in different stages (i.e., infants without teeth, preschool children with deciduous teeth, children with mixed teeth, adolescents with permanent teeth, and adults with permanent teeth). In older individuals, Proteobacteria was the dominant bacteria in deciduous dentition. In the mixed dentition and permanent dentition, the bacteriosis was dominated by Bacteroidetes. The abundance of Bacteroides Veillonella, Spirochaetes, and TM7 gradually increase with age [12]. Others [13] compared the oral microbial composition in different age groups from three days to 76 years. It was found that salivary microorganisms in permanent dentition (adolescence) was dominated by Phylum Firmicutes and that in deciduous dentition and permanent dentition (adult), Proteobacteria was the dominant bacteria, while Clostridium Prazmowski was more abundant in mixed dentition and permanent dentition (old age). Dental plaque and mucosal flora also showed changes with age and dentition replacement, suggesting that there is a change in microbial groups with age. 


\subsection{Individual Differences}

Studies have shown that marked differences exist in the composition of biofilms from person to person and from one type of intra-oral location to another and within the same individual. The saliva samples of 120 healthy people from 12 countries and regions were analyzed by one laboratory [14] and the results showed that there is significantly more variation among sequences from different individuals than among sequences from the same individual, but there is not significantly more variation among individuals from different locations than among individuals from the same location. Therefore, differences in the composition of genera among individuals are not structured by geography.

\subsection{Internal Relative Stability}

In the same individual, the differences at different times in oral microorganism groups were significantly lower than those in the intestinal tract, skin, and so on [15]. One research group [16] analyzed the saliva samples of healthy individuals at three different points in time in 29 days. It was found that the saliva microorganism group remained stable within five days, suggesting that the oral microorganism group had relative stability in a short period.

\section{Relationship between Oral Microorganism and Human Disease}

\subsection{Oral Microbiome and Oral Infection Diseases}

Oral infections are some of the most mutual diseases in humans. They mainly include caries, gingivitis, periodontitis, periapical periodontitis, and other infections of soft tissue. The majority of oral infectious diseases are non-specific infections, which is the result of oral microbes working together. According to statistic findings derived from studies, caries and periodontal disease are the two most common oral infections [17].

Dental caries (tooth decay) remains the most widespread chronic disease in both children and adults, even though it is largely preventable. Dental caries forms through a complex interaction over time between acid-producing bacteria and fermentable carbohydrate, and many host factors, including teeth and saliva. During the development of dental caries, the composition, structure, and function of oral microflora were significantly changed, and the distribution of various bacteria in the population of dental caries is significantly different from that of healthy populations.

Dental caries is the greatest common chronic disease of childhood and is the biggest unmet health care need among America's children. Periodontal disease is the utmost common infectious disease of adults. At least one-third of the populace is affected by chronic periodontitis, a bacterially induced destruction of the attachment of the tooth to the bone. Research is concentrated on understanding the etiology of these complex diseases and identifying new therapies. Other researchers are also interested in the interaction of oral pathogens with host tis- 
sues. More importantly, oral microorganism abnormalities can be detected before clinical caries symptoms appear. Left unchecked, hidden threats to oral health, such as gum disease, root cavities and infections, and oral cancer can lead to severe pain, loss of teeth, and serious health implications.

In one study, oral microbiota in 50 four-year-old children was tracked for two years. Before the clinical symptoms of early childhood caries appeared, the diversity of oral microbial $\alpha$ and $\beta$ increased significantly [10]. The composition of oral microorganisms is similar to that of dental caries patients, while it is different from that of healthy persons. Results showed that microbial Indicators of caries when de-trended for age, can predict the occurrence of dental caries for children.

The co-occurrence between oral floras of adult caries patients is significantly different from that of non-caries patients. Plaque microbiota structure exhibited a continuous gradient along with the first principal component, reflecting the transition from healthy to diseased states, which correlated with Mazza Gingival Index [18]. In the process of gingivitis, the transition of the microbiota was not a discrete process, but rather gradient-like and the degree of change is closely related to clinical symptoms. Furthermore, this change is reproduced whenever the same gingivitis occurs repeatedly. Therefore, plaque microbiota may provide advantages in predictive modeling of oral diseases [18].

Periapical periodontitis is an infectious disease occurring on the tooth periapical tissue, and it is the result of the interaction between the microbial community and the host immune response. With the development of gingivitis, the dominant bacteria in subgingival plaque gradually shift from Streptococcus to Actinomycetes, Capnocytophaga, Campylobacter, Eikenella, Fusobacterium, and Prevotella [19]. Besides, significant differences in the microbial functional gene structure were observed between healthy controls and periodontitis patients. The metabolic gene expression of individual species was also highly variable between patients.

Other oral diseases, such as periapical periodontitis [20] [21], halitosis [22], oral lichen planus [23] [24], also showed significant differences in oral microbial composition compared with healthy people. The dominant bacteria found in the tongue dorsum of patients within extraoral halitosis include Solobacteriummoorei, Atopobiumparvulum, and Eubacterium sulci. The major pathogenic factor of periapical periodontitis is microbial colonization in the root canal system. The most represented, abundant, and prevalent phyla in infected root canals were Proteobacteria, Firmicutes, Bacteroidetes, Fusobacteria, and Actinobacteria at the phylum level and Olsenellauli, Prevotella baroniae, Porphyromonas endodontalis, Fusobacterium nucleatum, and Tannerella forsythia at the species level [2].

\subsection{Oral Microbiome and Systemic Diseases}

In 1891, W. Miller published his theory on focal infection, indicating that microorganisms and/or their products can contact parts of the body adjacent to or distant from the mouth [25]. Subsequently, Dr. F. Billings speculated that infected teeth and tonsils could be considered responsible for several focal infec- 
tions such as arthritis, rheumatism, nephritis, endocarditis, and other unexplained diseases Billings [26]. The followers of this concept assume that microorganisms of dental plaque and their metabolic products may enter the bloodstream and result in many systemic and sometimes degenerative conditions.

In recent years, with the introduction and development of the concept of "periodontal medicine", it is generally agreed on that oral status is connected with systemic health, because poor oral health may occur concomitantly with more serious underlying diseases and/or it may predispose to other systemic diseases. Moreover, increasing evidence has supported that many systemic diseases are associated with disturbances in the oral ecosystem, such as diabetes, cardiovascular disease, preterm birth, and tumors rheumatoid arthritis (RA).

\subsubsection{Digestive System Disease}

Helicobacter pylori ( $H$. pylori) is a member of the oral microorganisms. Excluding the stomach, H. pylori in the oral cavity are found mostly in dental plaque. H. pylori exert numerous pathologic effects on the upper gastrointestinal tract including gastritis, peptic ulcer, gastric cancer, and mucosa-associated lymphoid tissue (MALT) lymphoma [27] [28].

Researchers [29] found that the antibacterial effect of $H$. pylori infectious gastritis was related to the colonization of $\mathrm{H}$. pylori in the oral cavity, suggesting that the health of the oral cavity would directly or indirectly affect the infection and reinfection of $H$. pylori in the stomach. Porphyromonas gingivalis is a resident oral bacteria group, which can lead to intestinal microbial community structure disorder and lead to the occurrence of intestinal inflammation [30] [31]. Another oral resident bacterium, Clostridium rubrum, is almost impossible to detect in the gut under normal conditions, but it can colonize the intestine in pathological conditions and play an important role in colorectal neoplasms and inflammatory bowel diseases [32] [33]. A variety of oral resident bacteria, such as Diplococcus, Micromonomonas, Clostridium, etc. can be detected in the appendix. Clostridium is closely related to the severity of appendicitis [34]. Some researchers have found that intestinal microbes in cirrhotic patients contain oral bacteria invasion, and these invasive oral microbes may be involved in the development of liver cirrhosis by promoting the over the reproduction of bacteria in the small intestine. By analyzing the 16s rRNA sequence of the fecal microbial community in patients with cirrhosis and liver cirrhosis complicated with hepatic encephalopathy, it was found that Streptococcus saliva could be translocated into the intestine of patients with liver cirrhosis and hyperpro-liferation. It is an important inducement of liver cirrhosis and liver cirrhosis complicated with hepatic encephalopathy [35]. In addition, poor oral health care behavior can change oral flora, cause intestinal microbial imbalance, and lead to inflammatory bowel disease [36].

\subsubsection{Oral Cancer}

It is found that there are specific microorganisms on the surface of cancerous 
tissues in oral cancer, and their composition is significantly different from that of normal mucosal microbes. Increasing evidence has indicated a role for oral microbiota in Oral squamous cell carcinoma (OSCC) through direct metabolism of carcinogens and inflammatory effects [37]. Others [37] found that oral cancer subjects had elevated counts of $C$. gingivalis, $P$. melaninogenica, and S. mitis in saliva related to OSCC-free individuals. These three bacteria were used as diagnostic markers. About $80 \%$ of oral squamous cell carcinoma can be diagnosed, so three kinds of bacteria have potential value as a diagnostic index of oral squamous cell carcinoma. Also, oral microorganisms may be involved in the development of distant organ tumors or affected by distant organ tumors. As an example, pancreatic cancer, the proportion of Granulicatella adiacens and S. mitis in salivary microorganism group was significantly lesser than that in healthy subjects [38].

\subsubsection{Cardiovascular Diseases}

Cardiovascular disease (CVD), involves multiple diseases with high mortality and includes all the diseases of the heart and circulation system including coronary heart disease, angina, heart attack, congenital heart disease, and stroke. There is now significant epidemiological evidence for an association of oral infections, particularly periodontitis and incident atherosclerotic CVD, which show that periodontitis is an important risk factor for the development of cardiovascular disease [39]. Anaeroglobus, closely related to periodontitis, can invade and colonize atherosclerotic plaques in patients [40]. In addition, Aggregatibater actinomycetemcomitans, Fusobacterium nucleatum, Campylobacter rectus, and Tannerella forsythia can be detected in almost all atherosclerotic plaques. Inflammation in the periodontium is proposed to increase the systemic inflammatory level of the host, which may influence plaque composition and rupture. A mechanism involving oral bacteria translocating into the bloodstream through inflamed gums has been proposed to affect atherogenesis. The overall microbial structure was similar in the normal population and atherosclerosis patients, but patients with indicative atherosclerosis had a higher relative abundance of Anaeroglobus than the normal population. Fak and coworkers [41] also found that Parvimonas associated positively with uCRP, Capnocytophaga, and Lactobacillus associated with blood lipid. In conclusion, the large quantity of Anaeroglobusin the oral cavity could be associated with symptomatic atherosclerosis [40] [42] [43]. The distribution of Fusobacterium, Streptococcus, and Neisseri is even associated with risk signals of the disease, such as total cholesterol and HDL. Bacteria associated with periodontosis may stimulate the production of inflammatory factors such as TNF $\alpha$, IL- $1 \beta$, IL- 6 by destroying body immunity, causing related inflammation and vascular endothelial damage, and promoting the formation of atherosclerotic plaques.

\subsubsection{Diabetes Mellitus}

Diabetes mellitus (DM) is a metabolic disease categorized by hyperglycemia due 
to insulin secretion deficiency or insulin dysfunction. There are two main types: type $1 \mathrm{DM}$ resulting from the body's failure to produce insulin and type $2 \mathrm{DM}$ resulting from insulin resistance. Both type 1 and type $2 \mathrm{DM}$ show a three- to four-fold increased risk of periodontitis, which is regarded as the sixth complication of DM [44]. It is generally thought that there is a bidirectional relationship between DM and periodontitis: Inflammatory mediators could negatively affect glycemic control, and increased glucose levels and resultant glycation end-products may alter the host response against bacterial infection [45]. The oral microbial community, as the initiating factor of periodontitis, is one of the causes of periodontal inflammation. In patients with periodontitis, complex interactions occur between oral microbes and the immune system, resulting in persistent chronic inflammation, and some oral microbes can also affect blood circulation. A large number of studies have shown that diabetes is associated with periodontitis: periodontitis leads to an increase in systemic inflammatory factors, increases in oxidative stress levels, and affects insulin sensitivity and blood glucose metabolism. Inflammation induced by periodontal microbe infection is probably one of the mechanisms that promote the development of DM. Some studies have shown that significant differences were observed in subgingival microbiota between diabetic and nondiabetic subjects. Compared with non-diabetic patients, subjects with diabetes and chronic periodontitis presented significant dissimilarities in subgingival biodiversity. Some phylotypes, such as Fusobacterium nucleatum, Veillonella parvula, V. dispar, and Eikenella corrodens, were noticed significantly more frequently in diabetic subjects than in nondiabetic subjects [46].

\subsubsection{Preterm Birth}

Preterm birth (PTB) is the second major direct cause of deaths in children younger than five years of age [47]. Risk of PTB is multifactorial, with intrauterine infection a major initiating factor. The composition of the placental microbial community of a preterm fetus and full-term fetus is different, which suggests that there may be an association between the placental microbial community and premature delivery. Researchers [48] analyzed 761 women delivering before the term of which 349 (46\%) presented with an intra-uterine infection. Bacteria belonging to a total of five phyla and 16 orders were found in the intra-uterine microbiota of the 349 pregnant women with intra-amniotic fluid. These detected microorganisms include not only reproductive tract pathogens but also respiratory pathogens and common oral microbes. There is an indication to support the hypothesis that bacterial infections at the orinasal cavity and the respiratory tract may be important reasons of preterm labor probably through the activation of abnormal inflammatory responses within the uterus and intrauterine tissues. The composition of the placental microbial community is most similar to that of oral microorganisms, compared with that of vaginal, intestinal, and respiratory sites [49].Confirmed by animal experiments, the microorganism samples of human saliva and subgingival plaque were injected into 
the tail vein of pregnant murine. Thus, all bacteria detected in the placenta originated from the starting material of pooled saliva or pooled subgingival plaque [50]. In addition, oral bacteria detected in amniotic fluid, such as Capnocytophaga, T. forsythensis, Treponema denticola, Td, Peptostreptococcus, Streptococcus sanguis, Aggregatibater actinomycetemcomitans, can indicate that oral bacteria may have pathological effects locally by invading amniotic fluid, leading to an increased risk of preterm delivery [51]. More research is needed to determine the causative relationship between PTB and adverse microbiome interactions.

\subsubsection{Rheumatoid Arthritis}

Rheumatoid arthritis (RA) is an autoimmune disease in which the body's immune system mistakenly attacks the joints. Several studies show that many similarities exist in the epidemiology and immunopathogenesis of periodontitis and RA, but the associations between their respective disease activities and severities are still uncertain. A prior investigation showed that the prevalence of the periodontal disease among individuals with RA was higher than the normal group. There are significant ecological disorders in oral microorganism in patients with RA [50]. This significant association between periodontitis and RA may be a reflection of a common underlying dysregulation of the inflammatory response in these individuals. Porphyromonas gingivitis and Actinobacillusactinomycetemcomitans can promote the progression of RA by their virulence factors [52].

\subsubsection{Alzheimer's Disease}

Alzheimer's disease (AD), also denoted to only as Alzheimer's, is a chronic neurodegenerative disease described by impairment of memory and finally by disturbances in reasoning, planning, language, and perception. It is the cause of $60 \%-70 \%$ of cases of dementia and usually starts slowly and worsens over time. As early as the 1990s, one group of researchers [53] suggested that oral microbes are closely related to the occurrence of AD. Researches, who used molecular and immunological techniques determined that oral Treponema may infect the brain via branches of the trigeminal nerve [54].The results strongly support a link between adverse oral bacteria and Alzheimer. Oral bacteria and their released endotoxin may activate microglia after invading the brain, leading to the discharge of pro-inflammatory cytokines such as TNF- $\alpha$ and IL-1. Therefore, exposure of brain tissue to high concentrations of TNF- $\alpha$ and IL-1 for a long time can make it easier for bacteria or endotoxin to infiltrate brain tissue and aggravate the clinical symptoms of the disease [55].

\section{The Role of the Oral Microbiome in Disease Prediction}

As an entrance to the digestive system, the oral cavity is a transportation hub connecting the human body inside and outside. Oral microorganisms are regulated by human health and disease state, so they can sensitively adjust these factors and amplify the preclinical symptoms. Also, oral microorganisms change in the state of disease with progressive, reproducible characteristics. The above 
characteristics of oral microorganism suggest that it has important value in early warning of disease risk and prognosis of curative effect.

As an example, traditional measures for dental caries risk assessment include oral bacteria count (e.g., Streptococcus mutans count and salivary Lactobacillus count), saliva biochemical analysis (saliva velocity, $\mathrm{pH}$ value, buffer capacity detection), past and previous dental caries, as well as personal oral hygiene (e.g., visible plaque levels), behavior (e.g., brushing habits), diet habits (e.g., sugar intake) or socioeconomic level, etc. [56]. The above-detailed evaluation of proximal factors seems to have only limited value for caries risk assessment, and elaborate tests involving bacterial counts or salivary analysis have only poor accuracy and, most likely, limited reliability [57] Most of these risk factors are subjective, susceptible to human preference and error, and are not acceptably reproducible among observers. For example, the inspection of baseline caries status typically depends on visual observation and the individual judgment of the examiner, making comparison across studies challenging. Personal data such as oral hygiene, frequency of food consumption, sugar consumption, and fluoride sources are usually collected voluntarily from patients and thus hard to quantitate accurately. Therefore, these models of dental caries risk assessment have not been widely used in clinical practice.

Tang and colleagues [58] developed a method called MiC (microbial indicators of caries) by high-throughput sequencing technology. This method is based on the discovery that oral flora changes preceded the clinical symptoms of caries. As the assembly of plaque or saliva is rapid and non-invasive and can be willingly performed at home, $\mathrm{MiC}$ can potentially serve as an objective, sensitive, and patient-friendly measure of gingivitis health diagnosis. Similarly, early childhood caries (ECC) susceptibility and preventive intervention of ECC and cross-study evaluation of oral care products in children populations are useful. Such methods can predict the occurrence of early caries in children with an accuracy rate of $81 \%$, and can effectively help doctors to carry out clinical intervention before the onset of the disease, to achieve the purpose of early prevention and early diagnosis [11].

At the same time, by monitoring the structural and functional changes of plaque flora during the stay-healthy mode, the caries-onset mode, and the caries-progression mode, a new method for the diagnosis of the severity of oral infection based on microorganisms was proposed, named "bacterial index of gingivitis, MiG". Compared with traditional methods, MiG was considered an objective, sensitive, and patient-friendly detection measure of gingivitis diagnosis [18].

In addition, changes in microbial composition are expected to reflect long-term outcomes. Proper cleaning of teeth and the use of mouthwash are routine clinical management in patients with gingivitis. Supragingival scaling [59], the brush-alone treatment, and the brush-plus-rinse treatment can relieve the clinical symptoms of the patients and achieve the goal of curing the disease by pushing 
the microbial group in the state of disease to return to the healthy state.

All of the three anti-gingivitis treatments may have distinguishable impacts on plaque microbiota, which could generate distinct "microbial signatures" for the treatment regimens. Dental scaling is widely measured as the most effective anti-gingivitis treatment for the most broad-spectrum and significant changes in plaque microbiota. However, for the brush-alone treatment, only the abundance of three oral taxa (Actinobaculum, TM7, and Leptotrichia) was significantly reduced [59].

Thus it can be seen; different treatments lead to not only a distinct temporal pattern of microbial composition changes during the reversal of gingivitis, but also the alteration of structural and functional consequences of oral microbiota. The results can reflect the dynamic changes of microflora in each treatment process and then predict the long-term clinical effect and guide the doctor to adjust the treatment plan to achieve the best clinical efficacy.

Also, the oral microbial community of patients with RA was significantly different from that of healthy persons [52]. This ecological disorder could be recovered through the treatment of RA, and the degree of recovery was closely related to the response of patients to treatment. According to the metagenome-wide association analysis of oral and intestinal microflora, the diagnostic accuracy of population classification model in differentiating healthy persons from patients with RA is close to $100 \%$, which suggested that oral the microbial community could be adjusted according to the recovery of body diseases and oral microbial community are highly sensitive to the occurrence, development and prognosis of systemic diseases.

Some of the breakthroughs in microbiologic-based treatments have also come from the oral mouth cavity. Within the repertoire of antibiotics accessible to a prescribing clinician, the common affect a broad range of microorganisms, as well as the normal flora. The ecological disruption resulting from antibiotic treatment regularly results in secondary infections or other negative clinical consequences. Eckert and colleagues [60] developed a new class of pathogen-selective molecules, called specifically targeted antimicrobial peptides (STAMPs), based on the union of a species-specific targeting peptide domain with a wide-spectrum antimicrobial peptide domain. In the current study, they concentrated on achieving the targeted killing of Streptococcus mutans, a cavity-causing bacterium that exists in a multispecies microbial community (dental plaque). Each STAMP consists of a nonspecific bactericidal module and a target binding module for a specific strain. Its working principle is that the target binding module binds the pathogen specifically and then kills the bacteria by the germicidal module connected with it. They also discovered that STAMPs constructed with peptides derived from competence-stimulating peptide (CSP) were strong against $S$. mutans grown in liquid or biofilm situations but did not affect additional oral streptococci tested [60]. The STAMPs presented here are clever of eliminating $S$. mutans from multispecies biofilms without affecting closely re- 
lated noncariogenic oral streptococci, signifying the potential of these molecules to be advanced into "probiotic" antibiotics which could selectively eliminate pathogens while preserving the protective benefits of healthy normal flora. This antimicrobial technique based on Streptococcus mutans establishes an important baseline for the design and preparation of STAMPs for other pathogens.

\section{Diagnostic and Prognostic Use of Saliva}

Over the last decade, sampling biologic compounds or biomarkers in saliva has garnered considerable research interest. As a diagnostic medium, use of saliva can be desirable over blood or serum, because it can be collected easily and noninvasively. Personnel training to assist in saliva collection can be minimum, and offers a cost-effective technique for large population screening. As noted by others [61] in clinically challenging situations, such as extracting samples from little children and handicapped or anxious elderly patients, saliva is a much more attractive alternative to venipuncture blood taking, particularly if taken several periods over time.

\subsection{Composition of Saliva}

Fluid found in the oral cavity contains mostly water and includes several inorganic and organic materials including proteins, hormones, enzymes, electrolytes, vitamins, and bacteria [48] [62]-[66]. Saliva is discharged into the oral cavity from glands. Major glands include the parotid, sublingual, submandibular, and minor glands include labial, buccal, lingual, and palatal [67] [68]. Secretions of these glands are associated with stimulation by the medulla [69]. Different amounts, flow rates of saliva, and the composition of saliva for each gland can vary and change throughout the day. Physiological and pathological situations can change saliva production. Odors and taste stimulate saliva flow, as well as chewing, hormonal status, drugs, age, and health status. Genetics and psychology can influence saliva secretions. Ultimately, the components found in saliva are blood-based and permeated into the oral cavity from different capillaries, acinar cells, and ductal cells (Holsinger and Bui, 2007). The basic secretory units of salivary glands are clusters of cells, termed acini. Such cells are defined as serious cells and secrete watery fluid. The functions of salivary components are to digest carbohydrates and lipids, food lubrication, destruction of bacteria, stimulate taste sensory receptors, maintains teeth enamel mineralization, buffer the acid components of food, antimicrobial functions and oral health [62] [63] [66]-[73].

\subsection{Saliva Biomarkers}

Usually, an individual can produce between 1 to 1.5 liters of saliva each day [74]. Collection of saliva is noninvasive, requires little to no training offering an appealing alternative to blood and serum extraction. Historically, systemic biomarkers in health correlate well between saliva and blood in diagnosis, e.g. HIV 
[75], hepatitis [76], oral cancer [77], periodontal disease [78] [79], and obesity [80]. The use of saliva is an increasing part of research with implications for basic and clinical purpose as a diagnostic armamentarium and an investigative tool for disease processes and disorders. Oxidative stress has been implicated in oral and systemic diseases. The use of monitoring oxidative stress biomarkers for diagnostic purposes is extremely attractive and actively being researched [81]. The usefulness of monitoring vitamin $\mathrm{C}$ and biomarkers of oxidative stress levels in subjects involved with exercise performance has recently been demonstrated [82]. However, there are limitations where saliva is not always the best specimen to represent systemic concentrations, e.g., amylase, phosphate and some proteomes [83] [84].

\subsection{Oral and Gut Microbiomes}

Microbial populations of the mouth and the gut are predictive of each other [84]. Although microbiomes of the oral cavity and gut are not the same, the changes in the gut are reflected in the oral microbiome [85] [86]. Regarding the composition of the microbiome, at the phylum level, the same phyla were reported as disturbed in saliva and fecal bacteria but differed in their order of abundance. Therefore, the implications are principles based on gut microbial description likely can apply to saliva. This has resulted in an upsurge of research interest in microbial fingerprints in saliva in various diseases. Systemic dysbiosis or pathologies associated with changes in the oral microbiome include irritable bowel diseases, colitis, celiac disease, obesity, leukemia, arthritis, and atherosclerosis [86]. Also, slight alterations in the oral microbiome could have systemic effects [85] [87]-[92]. Examples include; endothelial function, systemic inflammation, and glycemic control [93] [94].

\subsection{Best Practices in Using Saliva for Studying Biomarkers}

Saliva can be collected either under resting (unstimulated) or stimulated situations. Resting saliva is collected by draining the drool, spitting, using suction, or swabbing the mouth. For stimulation, subjects chew paraffin or have a dilute solution of citric acid applied to the tongue ( 0.1 to $0.2 \mathrm{~mol} / \mathrm{L})$. Collection accessories for saliva should be sterile and appropriate steps taken to prevent cross-contamination.

A disadvantage for using saliva is biomarkers may be at low concentrations, 100- to 1000 fold-lower compared to blood or plasma. Although traditional technological techniques are useful, such as ultra-concentration centrifugation, high-performance liquid chromatography, the postgenomic era provides opportunities for simple and parallel approaches to genomics and proteomics.

\section{Conclusion}

The oral microorganism group is the window of oral diseases and reflects gut microbiome and systemic diseases. Saliva is easy and noninvasive to collect and 
potentially an attractive fluid for a diagnostic and prognostic specimen. Community compositions are uniform across the gut and oral environment and seen by characteristic interspecies interaction among healthy subjects [95]. Also, microbiome composition at the phylum level is reported to be similar across the gut and oral microbiome; however, descending in their order of abundance [94]. As a model research system or as a surrogate to the gut microbiome, specimen from the oral cavity microbiome have many characteristics and advantages as noted in this review. Relationships between oral health and systemic diseases are quite profound, and future research about such relationships likely will be rewarding. The future of microbiome research is still early. The aim of future research should promote the application of microbiology in the field of stomatology by integrating information from medical technology, genome technology, and human microbial genome technology. Likewise, developing an understanding of the mechanistic underpinnings and the efficacy of microbes is yet to be revealed.

\section{Acknowledgements}

We thank the School of Public Health, Nanchang University, Jiangxi, China and School of Community Health Sciences, University of Nevada, Reno for making it possible for Zhongwei Zhang as a visiting scholar in the United States and Dr. Sher Todd for reading and editing this manuscript.

\section{Conflicts of Interest}

The authors declare no conflicts of interest regarding the publication of this paper.

\section{References}

[1] Dewhirst, F.E., Chen, T., Izard, J., Paster, B.J., Tanner, A.C.R., Yu, W.H., Lakshmanan, A. and Wade, W.G. (2010) The Human Oral Microbiome. Journal of Bacteriology, 192, 5002-5017. https://doi.org/10.1128/JB.00542-10

[2] Wade, W.G. (2013) The Oral Microbiome in Health and Disease. Pharmacological Research, 69, 137-143. https://doi.org/10.1016/j.phrs.2012.11.006

[3] Human Microbiome Project Consortium (2012) Structure, Function, and Diversity of the Healthy Human Microbiome. Nature, 486, 207-214. https://doi.org/10.1038/nature11234

[4] He, J.Z., Li, Y., Cao, Y.P., Xue, J. and Zhou, X.D. (2015) The Oral Microbiome Diversity and Its Relation to Human Diseases. Folia Microbiologica, 60, 69-80. https://doi.org/10.1007/s12223-014-0342-2

[5] Righolt, A.J., Jevdjevic, A.J., Marcenes, M. and Listl, S. (2018) Global-, Regional-, and Country-Level Economic Impacts of Dental Diseases in 2015. Journal of Dental Research, 97, 501-507. https://doi.org/10.1177/0022034517750572

[6] Chen, T., Yu, W.H., Izard, J., Baranova, O.V., Lakshmanan, A. and Dewhirst, F.E. (2010) The Human Oral Microbiome Database: A Web Accessible Resource for Investigating Oral Microbe Taxonomic and Genomic Information. Database ( $\mathrm{XX}^{-}$ ford), 2010, baq013. https://doi.org/10.1093/database/baq013 
[7] Keijser, B.J.F., Zaura, E., Huse, S.M., van der Vossen, M.B.M., Schuren, F.H.J., Montijn, R.C., Ten Cate, J.M. and Crielaard, W. (2008) Pyrosequencing Analysis of the Oral Microflora of Healthy Adults. Journal of Dental Research, 87, 1061-1070. https://doi.org/10.1177/154405910808701104

[8] Xu, X., He, J.Z., Xue, J., Wang, Y., Li, K., Zhang, K.K., Guo, Q., Liu, X.H., Zhou, Y., Cheng, L., Li, M.Y., Li, Y.Q., Li, Y., Shi, W.Y. and Zhou, X.D. (2015) Oral Cavity Contains Distinct Niches with Dynamic Microbial Communities. Environmental Microbiology, 17, 699-710. https://doi.org/10.1111/1462-2920.12502

[9] Huse, S.M., Ye, Y.Z., Zhou, Y.J. and Fodor, A.A. (2012) A Core Human Microbiome as Viewed Through 16S rRNA Sequence Clusters. PLoS ONE, 7, e34242. https://doi.org/10.1371/journal.pone.0034242

[10] Gizani, S., Papaioannou, W., Haffajee, A., Kavvadia, K., Quirynen, M. and Papagiannoulis, L. (2009) Distribution of Selected Cariogenic Bacteria in Five Different Intra-Oral Habitats in Young Children. International Journal of Pediatric Dentistry, 19, 193-200. https://doi.org/10.1111/j.1365-263X.2008.00956.x

[11] Dominguez-Bello, M.G., Costello, E.K., Contreras, M., Magris, M., Hidalgo, G., Fierer, N. and Knight, R. (2010) Delivery Mode Shapes the Acquisition and Structure of the Initial Microbiota across Multiple Body Habitats in Newborns. Proceedings of the National Academy of Sciences of the United States of America, 107, 11971-11975. https://doi.org/10.1073/pnas.1002601107

[12] Crielaard, W., Zaura, E., Schuller, A.A., Huse, S.M., Montijn, R.C. and Keijser, B.J.F. (2011) Exploring the Oral Microbiota of Children at Various Developmental Stages of Their Dentition in the Relation to Their Oral Health. BMC Medical Genomics, 4, 22. https://doi.org/10.1186/1755-8794-4-22

[13] Klimesova, K., Zakostelska, Z.J. and Tiaskalova-Hogenova, H. (2018) Oral Bacterial and Fungal Microbiome Impacts Colorectal Carcinogenesis. Frontiers in Microbiology, 9, 774. https://doi.org/10.3389/fmicb.2018.00774

[14] Nasidze, I., Li, J., Quinque, D., Tang, K. and Stoneking, M. (2009) Global Diversity in the Human Salivary Microbiome. Genome Research, 19, 636-643. https://doi.org/10.1101/gr.084616.108

[15] Costello, E.K., Lauber, C.L., Hamady, M., Fierer, N., Gordon, J.I. and Knight, R. (2009) Bacterial Community Variation in Human Baby Habitats across Space and Time. Science, 326, 1694-1697. https://doi.org/10.1126/science.1177486

[16] Lazarevic, V., Whiteson, K., Hernandez, D., François, P. and Schrenzel, J. (2010) Study of Inter- and Intra-Individual Variations in the Salivary Microbiota, BMC Genomics, 11, 523. https://doi.org/10.1186/1471-2164-11-523

[17] Lopez, R., Smith, P.C., Gostemeyer, G. and Schwendicke, F. (2017) Ageing, Dental Caries, and Periodontal Diseases. Journal of Clinical Periodontology, 44, S145-S152. https://doi.org/10.1111/jcpe.12683

[18] Huang, S., Li, R., Zeng, X.W., He, T., Zhao, H.L., Chang, A., Bo, C.P., Chen, J., Yang, F., Knight, R., Liu, J.Q., Davis, C. and Xu, J. (2014) Predictive Modeling of Gingivitis Severity and Susceptibility via Oral Microbiota. The ISME Journal, 8, 1768-1780. https://doi.org/10.1038/ismej.2014.32

[19] Li, Y., He, Z.L., Zhou, Y., Yuan, M.T., Xu, X., Sun, F.F., Liu, C.C., Li, J.Y., Xie, W.B., Deng, Y. and Qin, Y.J. (2014) Phylogenetic and Functional Gene Structure Shifts of the Oral Microbiomes in Periodontitis Patients. The ISME Journal, 8, 1879-1891. https://doi.org/10.1038/ismej.2014.28

[20] Siqueira Jr., J.F. and Rôças, I.N. (2009) Community as the Unit of Pathogenicity: An Emerging Concept as to the Microbial Pathogenesis of Apical Periodontitis. Oral 
Surgery, Oral Medicine, Oral Pathology, Oral Radiology, and Endodontology, 107, 870-878. https://doi.org/10.1016/j.tripleo.2009.01.044

[21] Siqueira Jr., J.F. (2002) Endodontic Infections: Concepts, Paradigms, and Perspectives. Oral Surgery, Oral Medicine, Oral Pathology, Oral Radiology, and Endodontology, 94, 281-293. https://doi.org/10.1067/moe.2002.126163

[22] Kazor, C.E., Mitchell, P.M., Lee, A.M., Stokes, L.N., Loesche, W.J., Dewhirst, F.E. and Paster, B.J. (2003) Diversity of Bacterial Populations on the Tongue Dorsa of Patients with Halitosis and Healthy Patients. Journal of Clinical Microbiology, 41, 558-563. https://doi.org/10.1128/JCM.41.2.558-563.2003

[23] Wang, K., Lu, W.X., Tu, Q.C., Ge, Y.C., He, J.Z., Zhou, Y., Gou, Y.P., Van Nostrand, J.D., Qin, Y.J., Li, J.Y., Zhou, J.Z., Li, Y., Xiao, L.Y. and Zhou, X.D. (2016) Preliminary Analysis of Salivary Microbiome and Their Potential Roles in Oral Lichen Planus. Scientific Reports, 6, Article No. 22943.

https://doi.org/10.1038/srep22943

[24] Wang, K., Miao, T.Y., Lu, W.X., He, J.Z., Cui, B.M., Li, J.Y., Li, Y. and Xiao, L.Y. (2015) Analysis of Oral Microbial Community and Th17-Associated Cytokines in Saliva of Patients with Oral Lichen Planus. Microbiology and Immunology, 59, 105-113. https://doi.org/10.1111/1348-0421.12232

[25] Miller, W. (1891) The Human Mouth as a Focus of Infection. Dental Cosmos, 36, 689-713. https://doi.org/10.1016/S0140-6736(02)01387-9

[26] Billings, F. (1914) Focal Infection: Its Broader Application in the Etiology of Disease. JAMA, 9, 899-903. https://doi.org/10.1001/jama.1914.02570110001001

[27] Israel, D.A. and Peek Jr., R.M. (2006) The Role of Persistence in Helicobacter pylori Pathogenesis. Current Opinion in Gastroenterology, 22, 3-7. https://doi.org/10.1097/01.mog.0000194790.51714.f0

[28] Konturek, P.C., Konturek, S.J., Starzyska, T., et al. (2000) Helicobacter pylori Gastrin Link in MALT Lymphoma Aliment. Pharmacology \& Therapeutics, 14, 1311-1318. https://doi.org/10.1046/j.1365-2036.2000.00832.x

[29] Miyabayashi, H., Furihata, K., Shimizu, T., Ueno, I. and Akamatsu, T. (2000) Influence of Oral Helicobacter pylori on the Success of Eradication Therapy against Gastric Helicobacter pylori. Helicobacter, 5, 30-37. https://doi.org/10.1046/j.1523-5378.2000.00004.x

[30] Nakajima, M., Arimatsu, K., Kato, T., Matsuda, Y., Minagawa, Takahashi, N., Ohno, H. and Yamazaki, K. (2015) Oral Administration of Pgingivalis Induces Dysbiosis of Gut Microbiota and Impaired Barrier Function Leading to Dissemination of Enterobacteria to the Liver. PLOS ONE, 10, e0134234.

https://doi.org/10.1371/journal.pone.0134234

[31] Arimatsu, K., Yamada, H., Miyazawa, H., Minagawa, T., Nakajima, M., Ryder, M.I., Gotoh, K., Motooka, D., Nakamura, S., Iida, T. and Yamazaki, K. (2014) Oral Pathobiont Induces Systemic Inflammation and Metabolic Changes Associated with Alteration of Gut Microbiota. Scientific Reports, 4, Article No. 4828. https://doi.org/10.1038/srep04828

[32] Warren, R.L., Freeman, D.J., Pleasance, S., Watson, P., Moore, R.A., Cochrane, K., Allen-Vercoe, E. and Holt, R.A. (2013) Co-Occurrence of Anaerobic Bacteria in Colorectal Carcinomas. Microbiome, 1, 16. https://doi.org/10.1186/2049-2618-1-16

[33] Bashir, A., Miskeen, A.Y., Bhat, A., Fazili, K.M. and Ganai, B.A. (2015) Fusobacterium nucleatum: An Emerging Bug in Colorectal Tumorigenesis. European Journal of Cancer Prevention, 24, 373-385. https://doi.org/10.1097/CEJ.0000000000000116 
[34] Guinane, C.M., Tadrous, A., Fouhy, F., Ryan, C.A., Dempsey, E.M., Murphy, B., Andrews, E., Cotter, P.D., Stanton, C. and Ross, R.P. (2013) Microbial Composition of Human Appendices from Patients Following Appendectomy. mBio, 4, e00366-12. https://doi.org/10.1128/mBio.00366-12

[35] Zhang, Z.G., Zhai, H.Q., Geng, J.W., Yu, R., Ren, H.Q., Fan, H. and Shi, P. (2013) Large-Scale Survey of Gut Microbiota Associated with MHE via 16S rRNA-Based Pyrosequencing. The American Journal of Gastroenterology, 108, 1601-1611. https://doi.org/10.1038/ajg.2013.221

[36] Singhal, S., Dian, D., Keshavarzian, A., Fogg, L., Fields, J.Z. and Farhadi, A. (2011) The Role of Oral Hygiene in Inflammatory Bowel Disease. Digestive Diseases and Sciences, 56, 170-175. https://doi.org/10.1007/s10620-010-1263-9

[37] Mager, D.L., Haffajee, A.D., Devlin, P.M., Norris, C.M., Posner, M.R. and Goodson, J.M. (2005) The Salivary Microbiota as a Diagnostic Indicator of Oral Cancer: A Descriptive, Non-Randomized Study of Cancer-Free and Oral Squamous Cell Carcinoma Subjects. Journal of Translational Medicine, 3, 27-29.

https://doi.org/10.1186/1479-5876-3-27

[38] Farrell, J.J., Zhang, L., Zhou, H., Chia, D., Elashoff, D., Akin, D., Paster, B.J., Joshipura, K. and Wong, D.T.W. (2012) Ariations of Oral Microbiota Are Associated with Pancreatic Diseases Including Pancreatic Cancer. Gut, 61, 582-588. https://doi.org/10.1136/gutjnl-2011-300784

[39] Dietrich, T., et al. (2013) The Epidemiological Evidence behind the Association between Periodontitis and Incidental Hero Sclerotic Cardiovascular Disease. Journal of Periodontology, 84, 70-84. https://doi.org/10.1902/jop.2013.134008

[40] Ford, P.J., Gemmell, E., Hamlet, S.M., Hasan, A., Walker, P.J., West, M.J., Cullinan, M.P. and Seymour, G.J. (2005) Cross-Reactivity of GroEL Antibodies with Human Heat Shock Protein 60 and Quantification of Pathogens in Atherosclerosis. Oral Microbiology and Immunology, 20, 296-302. https://doi.org/10.1111/j.1399-302X.2005.00230.x

[41] Fak, F., Tremaroli, V., Bergstrom, G. and Backhed, F. (2015) Oral Microbiota in Patients with Atherosclerosis. Atherosclerosis, 243, 573-578. https://doi.org/10.1016/j.atherosclerosis.2015.10.097

[42] Figuero, E., Sánchez-Beltrán, M., Cuesta-Frechoso, S., Tejerina, J.M., del Castro, J.A., Gutiérrez, J.M., Herrera, D. and Sanz, M. (2011) Detection of Periodontal Bacteria in Atheromatous Plaque by Nested Polymerase Chain Reaction. Journal of Periodontology, 82, 1469-1477. https://doi.org/10.1902/jop.2011.100719

[43] Pucar, A., Milasin, J., Lekovic, V., Vukadinovic, M., Ristic, M., Putnik, S. and Kenney, E.B. (2007) Correlation between Atherosclerosis and Periodontal Putative Pathogenic Bacterial Infections in Coronary and Internal Mammary Arteries. Journal of Periodontology, 78, 677-682. https://doi.org/10.1902/jop.2007.060062

[44] Loe, H. (1993) Periodontal Disease: The Sixth Complication of Diabetes Mellitus. Diabetes Care, 16, 329-334. https://doi.org/10.2337/diacare.16.1.329

[45] Diaz-Romero, R.M. and Ovadia, R. (2007) Diabetes and Periodontal Disease: A Bidirectional Relationship. Biology and Medicine, 14, 6-9.

[46] Ohlrich, E.J., Cullinan, M.P. and Leichter, J.W. (2010) Diabetes, Periodontitis, and the Subgingival Microbiota. Journal of Oral Microbiology, 2. https://doi.org/10.3402/jom.v2i0.5818

[47] Berkovitz, B.K.B., Holland, G.R. and Moxham, B.J. (2002) Oral Anatomy, Histology, and Embryology. 3rd Edition, Mosby: New York.

[48] Mendz, G.L., Kaakoush, N.O. and Quinlivan, J.A. (2013) Bacterial Etiological 
Agents of Intra-Amniotic Infections and Preterm Birth in Pregnant Women. Frontiers in Cellular and Infection Microbiology, 3, 58-60. https://doi.org/10.3389/fcimb.2013.00058

[49] Offenbacher, S., Katz, V., Fertik, G., Collins, J., Boyd, D., Maynor, G., McKaig, R., and Beck, J. (1996) Periodontal Infection as a Possible Risk Factor for Preterm Low Birth Weight. Journal of Periodontology, 67, 1103-1113.

https://doi.org/10.1902/jop.1996.67.10s.1103

[50] Bartold, P.M., Marshall, R.I. and Haynes, D.R. (2005) Periodontitis and Rheumatoid Arthritis: A Review. Journal of Periodontology, 76, 2066-2074. https://doi.org/10.1902/jop.2005.76.11-S.2066

[51] Fardini, Y., Chung, P., Dumm, R., Joshi, N. and Han, Y.W. (2010) Transmission of Diverse Oral Bacteria to Murine Placenta: Evidence for the Oral Microbiome as a Potential Source of Intrauterine Infection. Infection and Immunity, 78, 1789-1796. https://doi.org/10.1128/IAI.01395-09

[52] Zhang, X., Zhang, D.Y., Jia, H.J., Feng, Q., Wang, D.H., Liang, D., et al. (2015) The Oral and Gut Microbiomes Are Perturbed in Rheumatoid Arthritis and Partly Normalized after Treatment. Nature Medicine, 21, 895-905.

https://doi.org/10.1038/nm.3914

[53] Miklossy, J. (1994) Alzheimer Disease-A Spirochetosis? In: Giacobini, E. and Becker, R.E., Eds., Advances in Alzheimer Disease, Springer, Birkhäuser, Boston, 41-45. https://doi.org/10.1007/978-1-4615-8149-9_7

[54] Riviere, G.R., Riviere, K.H. and Smith, K.S. (2002) Molecular and Immunological Evidence of Oral Treponema in the Human Brain and Their Association with Alzheimer's Disease. Oral Microbiology and Immunology, 17, 113-118. https://doi.org/10.1046/j.0902-0055.2001.00100.x

[55] Dickstein, J.B., Moldofsky, H. and Hay, J.B. (2000) Brain-Blood Permeability: TNF- $\alpha$ Promotes Escape of Protein Tracer from CSF to Blood. American Journal of Physiology_Regulatory, Integrative and Comparative Physiology, 7, R148-R151. https://doi.org/10.1152/ajpregu.2000.279.1.R148

[56] Mejàre, I., Axelsson, S., Dahlén, G., Espelid, I., Norlund, A., Tranæus, S. and Twetman, S. (2014) Caries Risk Assessment. A Systematic Review. Acta Odontologica Scandinavica, 72, 81-91. https://doi.org/10.3109/00016357.2013.822548

[57] Tellez, M., Gomez, J., Pretty, I., Ellwood, R. and Smail, A.I. (2013) Evidence on Existing Caries Risk Assessment Systems: Are They Predictive of Future Caries? Community Dentistry and Oral Epidemiology, 41, 70-78. https://doi.org/10.1111/cdoe.12003

[58] Teng, F., Yang, F., Huang, S., Bo, C.P., Xu, Z.Z., Amir, A., Knight, R., Ling, J.Q. and $\mathrm{Xu}$, J. (2015) Prediction of Early Childhood Caries via Spatial-Temporal Variations of Oral Microbiota. Cell Host \& Microbe, 18, 96-306. https://doi.org/10.1016/j.chom.2015.08.005

[59] Huang, S., Li, Z., He, T., Bo, C.P., Chang, J.L., Li, L., He, Y.Y., Liu, J.Q., Charbonneau, D., Li, R. and Xu, J. (2016) Microbiota-Based Signature of Gingivitis Treatments: A Randomized Study. Scientific Reports, 6, Article No. 24705. https://doi.org/10.1038/srep24705

[60] Eckert, R., He, J., Yarbrough, D.K., Qi, F.X., Anderson, M.H. and Shi, W.Y. (2006) Targeted Killing of Streptococcus mutants by a Pheromone-Guided "Smart" Antimicrobial Peptide. Antimicrobial Agents and Chemotherapy, 50, 3651-3657. https://doi.org/10.1128/AAC.00622-06 
[61] Pfaffe, T., Copper-White, J., Beyerlein, P., Kostner, K. and Punyadeera, C. (2011) Diagnostic Potential of Saliva: Current State and Future Applications. Clinical Chemistry, 57, 675-687. https://doi.org/10.1373/clinchem.2010.153767

[62] Edgar, W.M. (1992) Saliva: Its Secretion, Composition, and Functions. British Dental Journal, 172, 305-312. https://doi.org/10.1038/sj.bdj.4807861

[63] Zalewska, A., Zwierz, K., Zólkowski, K. and Gindzienski, A. (2000) Structure and Biosynthesis of Human Salivary Mucins. Acta Biochimica Polonica, 47, 1067-1079.

[64] Van NieuwAmerongen, A., Bolscher, J.G. and Veerman, E.C. (2004) Salivary Proteins: Protective and Diagnostic Value in Cardiology? Caries Research, 38, 247-253. https://doi.org/10.1159/000077762

[65] Humphrey, S.P. and Williamson, R.T. (2001) A Review of Saliva: Normal Composition, Flow, and Function. Journal of Prosthetic Dentistry, 85, 162-169. https://doi.org/10.1067/mpr.2001.113778

[66] Ahmadi-Motamayel, F., Falsafi, P., Goodarzi, M.T. and Poorolajal, J. (2016) Evaluation of Salivary Catalase, Vitamin C, and Alpha-Amylase in Smokers and Non-Smokers: A Retrospective Cohort Study. Journal of Oral Pathology \& Medicine, 16, 317-321. https://doi.org/10.18295/squmj.2016.16.03.009

[67] Forde, M.D., Koka, S., Eckert, S.E., Carr, A.B. and Wong, D.T. (2006) Systemic Assessments Utilizing Saliva: Part 1 General Consideration and Current Assessments. The International Journal of Prosthodontics, 19, 43-52.

[68] Holmberg, K.V. and Hoffman, M.P. (2014) Anatomy, Biogenesis, and Regeneration of Salivary Gland. Monographs in Oral Science, 24, 1-13. https://doi.org/10.1159/000358776

[69] Grant, D.A., Stern, I.B. and Listgarten, M.A. (1988) Periodontics. 6th Edition, CV Mosby, St. Louis, 135-146.

[70] Holsinger, F. and Bui, D. (2007) Salivary Gland Disorders. Springer, Berlin.

[71] De Almedia, P., Del, V., Gregio, A.M., Machado, M.A., de Lima, A.A. and Azevedo, L.R. (2008) Saliva Composition and Functions: A Comprehensive Review. The Journal of Contemporary Dental Practice, 122, 72-80.

[72] Pedersen, A.M., Bardo, A., Jensen, S.B. and Nauntofte, B. (2002) Saliva and Gastrointestinal Functions of Taste, Mastication, Swallowing, and Digestion. Oral Diseases, 8, 117-129. https://doi.org/10.1034/j.1601-0825.2002.02851.x

[73] Mese, H. and Matsuo, R. (2007) Salivary Secretion, Taste and Hypo Salivation. Journal of Oral Rehabilitation, 34, 711-723. https://doi.org/10.1111/j.1365-2842.2007.01794.x

[74] Edgar, W. (1990) Saliva and Dental Health. Clinical Implications of Saliva: Report of a Consensus Meeting. British Dental Journal, 169, 96-98.

https://doi.org/10.1038/sj.bdj.4807284

[75] Delaney, K.P., Branson, B.M., Uniyal, A., Kerndt, P.R., Keenan, P.A., Jafa, K., Gardner, A.D., Jamieson, D.J. and Bulterys, M. (2006) Performance of an Oral Fluid Rapid HIV-1/2 Test: Experience from Four CDC Studies. AIDS, 20, 1655-1660. https://doi.org/10.1097/01.aids.0000238412.75324.82

[76] Amado, L.A., Villar, L.M., de Paula, V.S., de Almeida, A.J. and Gaspar, A.M. (2006) Detection of Hepatitis A, B, and C Virus-Specific Antibodies Using Oral Fluid for Epidemiological Studies. Memórias do Instituto Oswaldo Cruz, 101, 149-155. https://doi.org/10.1590/S0074-02762006000200006

[77] Mage, D.L., Haffajee, A.D., Devlin, P.M., Norris, C.M., Posner, M.R. and Goodson, J.M. (2005) The Salivary Microbiota as a Diagnostic Indicator of Oral Cancer: A 
Descriptive, Non-Randomized Study of Cancer-Free and Oral Squamous Cell Carcinoma Subjects. Journal of Translational Medicine, 27, 235-240. https://doi.org/10.1186/1479-5876-3-27

[78] Paju, S., Pussinen, P.J., Suominen-Taipale, L., Hyvönen, M., Knuuttil, M. and Könönen, E. (2009) Detection of Multiple Pathogenic Species in Saliva Is Associated with Periodontal Infection in Adults. Journal of Clinical Microbiology, 47, 235-238. https://doi.org/10.1128/JCM.01824-08

[79] Paster, B.J. and Dewhirst, F.E. (2009) Molecular Microbial Diagnosis. Periodontology, 51, 38-44. https://doi.org/10.1111/j.1600-0757.2009.00316.x

[80] Goodson, J.M., Groppo, D., Halem, S. and Carpino, E. (2009) Is Obesity an Oral Bacterial Disease? Journal of Dental Research, 8, 519-523. https://doi.org/10.1177/0022034509338353

[81] Al-Rawi, N.H. (2012) Diabetes, Oxidative Stress, Antioxidants and Saliva: A Review. In: Lushchak, V.I. and Gospodaryov, D.V., Eds., Oxidative Stress Diseases, IntechOpen, London, Chapter 13.

https://www.intechopen.com/books/oxidative-stress-and-diseases/diabetes-oxidativ e-stress-antioxidants-and-saliva

[82] Evans, L.W. and Omaye, S.T. (2017) Use of Saliva Biomarkers to Monitor Efficacy of Vitamin C in Exercise-Induced Oxidative Stress. Antioxidants, 6, 5. https://doi.org/10.3390/antiox6010005

[83] Wang, J., Schipper, H.M., Velly, A.M., Mohit, S. and Gornitsky, M. (2015) Salivary Biomarkers of Oxidative Stress: A Critical Review. Free Radical Biology \& Medicine, 85, 95-104. https://doi.org/10.1016/j.freeradbiomed.2015.04.005

[84] Din, T. and Schloss, P.D. (2014) Dynamics and Associations of Microbial Community Types across the Human Body. Nature, 509, 357-360. https://doi.org/10.1038/nature13178

[85] Rautava, J., Pinnell, L.J., Vong, L., Akseer, N., Assa, A. and Sherman, P.M. (2014) Oral Microbiome Composition Changes in Mouse Model of Colitis. Journal of Gastroenterology and Hepatology, 2, 521-527.

[86] Docktor, M.J., Paster, B.J., Abramowica, S., Ingram, J., Wang, Y.E., Correll, M., Jiang, H., Cotton, S.L., Korkaras, A.S. and Bousvaros, A. (2012) Alterations in Diversity of the Oral Microbiome in Pediatric Inflammatory Bowel Disease. Inflammatory Bowel Disease, 18, 935-942. https://doi.org/10.1002/ibd.21874

[87] Said, H.S., Sunda, W., Nakagome, S., Chinen, H., Oshima, K., Kim, S., Dimura, R., Iraha, I.H. and Fujita, J. (2014) Dysbiosis of Salivary Microbiota in Inflammatory Bowel Disease and Its Association with Oral Immunological Biomarkers. DNA Research, 21, 15-25. https://doi.org/10.1093/dnares/dst037

[88] Wang, Y., Xue, J., Zhou, X., You, M., Du, Q., Yang, X., He, J., Zou, J., Cheng, L. and Li, M. (2014) Oral Microbiota Distinguishes Acute Lymphoblastic Leukemia Pediatric Hosts from Healthy Populations. PLoS ONE, 9, e102116.

https://doi.org/10.1371/journal.pone.0102116

[89] Woff, B., Berger, T., Frese, C., Max, R., Blank, N., Lorenz, H.M. and Wolff, D. (2014) Oral Status in Patients with Early Rheumatoid Arthritis: A Prospective, Case-Control Study. Rheumatology, 53, 562-531. https://doi.org/10.1093/rheumatology/ket362

[90] Yeoh, N., Burton, J.P., Suppiah, P., Reid, G. and Stebbing, S. (2013) The Role of the Microbiome in Rheumatic Diseases. Current Rheumatology Reports, 15, 12-14. https://doi.org/10.1007/s11926-012-0314-y

[91] Koren, O., Spor, A., Feli, J., Fak, F., Stombaugh, J., Tremaroli, V., Behre, C.J., 
Knight, R., Fagerberg, B. and Ley, R.E. (2011) Human Oral, Gut, and Plaque Microbiota in Patients with Atherosclerosis. Proceedings of the National Academy of Sciences of the United States of America, 108, 4592-4598.

https://doi.org/10.1073/pnas.1011383107

[92] Li, J., Quinque, D., Li, M., Horz, H.P., Andre, C., et al. (2013) The Saliva Microbiome of Pan and Homo. BMC Microbiology, 13, 204. https://doi.org/10.1186/1471-2180-13-204

[93] Major, G. and Spiller, R. (2014) Irritable Bowel Syndrome, Inflammatory Bowel Disease, and the Microbiome. Current Opinion in Endocrinology, Diabetes and Obesity, 21, 15-21. https://doi.org/10.1097/MED.0000000000000032

[94] Teeuw, W.J., Gerdes, V.E. and Loss, B.G. (2010) Effect of Periodontal Treatment on Glycemic Control of Diabetic Patients: A Systematic Review and Meta-Analysis. Diabetes Care, 33, 421-427. https://doi.org/10.2337/dc09-1378

[95] Bashan, A., Gibson, T.E., Friedman, J., Casey, V.J., Weiss, S.T., Hohman, E.L., et al. (2016) University of Human Microbial Dynamics. Nature, 535, 259-262. https://doi.org/10.1038/nature18301 